\title{
Incidence of and Risk Factors for Lactational Mastitis: A Systematic Review
}

Journal of Human Lactation 2020, Vol. 36(4) 673-686 (C) The Author(s) 2020

Article reuse guidelines: sagepub.com/journals-permissions DOI: I0.1 I 77/0890334420907898 journals.sagepub.com/home/jhl

(S)AGE

\author{
Emily Wilson, MSc ${ }^{1,2}$, Susannah L Woodd, MSc' ${ }^{1}$, \\ and Lenka Benova, PhD ${ }^{1,3}$ (D)
}

\begin{abstract}
Background: Lactational mastitis is a maternal morbidity that affects the wellbeing of women and their babies, including through breastfeeding discontinuation.

Research Aim: To systematically review the available global literature on the frequency of lactational mastitis, and to summarize the evidence on risk factors for lactational mastitis. We also describe gaps in the evidence and identify priority areas for future research.

Methods: We systematically searched and screened 6 databases and included 26 articles, conducted meta-analysis of disease frequency, and narratively synthesized evidence on risk factors.

Results: In II (42\%) articles researchers reported a measure of disease frequency; 5 (I9\%) reported risk factors, and I0 (39\%) included both. Overall, the quality of studies was low, related to suboptimal measurement of disease frequency, high risk of bias, reverse causality, and incomplete adjustment for confounding. Meta-analysis was based on 3 studies (pooled incidence between birth and Week 25 postpartum: II.I episodes per I,000 breastfeeding weeks; 95\% Cl [I0.2-I2.0]); with high heterogeneity across contexts and highest incidence in the first four weeks postpartum. Researchers assessed 42 potential risk factors; nipple damage was the most frequently studied and strongly associated with mastitis. There was a scarcity of studies from low-resource settings.

Conclusions: Lactational mastitis is a common condition, but the wide variability in incidence across contexts suggested that a substantial portion of this burden might be preventable. Provision of care to breastfeeding women at risk for or affected by mastitis is currently constrained due to a critical lack of high quality epidemiological evidence about its incidence and risk factors.
\end{abstract}

\section{Keywords}

breastfeeding, breastfeeding difficulties, breast pain, epidemiological methods, mastitis

\section{Background}

Lactational mastitis is defined as inflammation of the breast tissue and is commonly experienced by breastfeeding women (Amir et al., 2007). It is a painful condition with high fever; flu-like symptoms, for example aches and chills; and red, tender, hot, and swollen areas of the breast (Lawrence, 1989; World Health Organization, 2000). It is diagnosed symptomatically and there is no broadly accepted clinical definition (Zarshenas et al., 2017). Mastitis can be experienced on a continuum from mild inflammation to more severe disease (Michie et al., 2003). There is also no consensus on the aetiology, which may be inflammatory, infectious, based on a bacterial imbalance, or multifactorial (Baeza, 2016). Human milk is colonized by a large variety of bacteria, some of which might originate from the maternal gut via an endogenous pathway (Marín, 2017). These commensal organisms appear important for the developing infant gut microbiome.
Potentially pathogenic bacteria have been isolated from the human milk of healthy breastfeeding women although there is evidence that certain bacteria, in particular Staphylococcus aureus, are more common in women with mastitis than those without (Hager et al. 1996; Kvist et al., 2008). Aetiological theories include bacterial infection, for example via cracked nipples (Foxman et al., 2002) or a dysbiotic process where some species outgrow, while others disappear (Delgado,

\footnotetext{
'London School of Hygiene and Tropical Medicine, London, UK

${ }^{2}$ University College London, University College, London, UK

${ }^{3}$ Department of Public Health, Institute of Tropical Medicine, Antwerp, Belgium

Date submitted: January 18, 2019; Date accepted: January 30, 2020.

Corresponding Author:

Lenka Benova PhD, Department of Public Health, Institute of Tropical Medicine, Nationalestraat I55, Antwerp, Antwerp 2000 Belgium.

Email: Ibenova@itg.be
} 
2008). In addition, virulence factors, production of biofilm, antimicrobial resistance, and interaction with the host immune system have all been proposed to play a part (Contreras, 2011).

Lactational mastitis may be clinically described as "selflimiting" as it usually resolves without medical intervention through self-management, for example massaging the affected breast, feeding or expressing frequently enough to empty the affected breast, and using cold compresses to soothe the inflammation (Spencer, 2008; Wambach, 2003). However, some women require antibiotics to treat infection and, if left under- or un-treated, infective mastitis can lead to breast abscess or septicemia, which may necessitate hospitalization and possibly surgery (Thomsen et al., 1984). It is possible to develop mastitis more than once and women might experience lactational mastitis multiple times while breastfeeding the same child. Women who develop mastitis may cease breastfeeding prematurely due to the pain the condition causes, fear that antibiotics may pass through the milk, or incorrect advice from health professionals to cease breastfeeding (Foxman et al., 2002). This can put infants at risk of infection, as well as increasing their chance of obesity and metabolic disease later in life, particularly in low income settings where there is a high burden of disease and low access to clean water and sanitation (Dieterich et al., 2013). Therefore, mastitis not only puts the mother at risk for more serious health complications but may also lead to potential loss in health benefits for the infant (Wambach, 2003). A recent systematic review of systematic reviews on maternal morbidity found that no systematic reviews on the frequency of mastitis have been published (Gon et al., 2018). The aim of this study is to (1) systematically review the available global literature on the frequency of lactational mastitis, and to (2) summarize the evidence on risk factors for lactational mastitis.

\section{Methods}

\section{Design}

We conducted a systematic review of the literature to provide an exhaustive summary of the evidence on frequency and risk factors of lactational mastitis globally to address the study aims.

\section{Sample}

We searched for primary studies with a measure of the frequency of lactational mastitis or evidence for any risk factor of lactational mastitis. Numbers of identified and retained articles are presented in a flow diagram (Figure 1). We included all studies of human lactational mastitis regardless of the definition of the condition and irrespective of follow up time since birth. Articles were included in full-text screening if they reported the results of primary or secondary research (i.e., not a review or opinion piece), estimated

\section{Key Messages}

- Lactational mastitis is a painful condition that can lead to discontinuation of breastfeeding, but its frequency and risk factors have not been systematically summarized.

- We found that lactational mastitis is common, affecting approximately one in four women breastfeeding during the first 26 weeks postpartum. However, the incidence of lactational mastitis varied widely across settings.

- Among the 42 unique risk factors assessed by researchers, nipple damage seemed to be most consistently associated with mastitis.

the disease burden of or risk factors for lactational mastitis, and were in English. Studies not conducted in humans were excluded.

The initial search returned 6,562 references. After deduplication, 3,807 unique references were screened by title and abstract and 46 articles were selected for full text screening (Figure 1). We note the substantial number $(>$ 2,800 ) of articles that were excluded due to the study population being non-human (predominantly cows, but also goats, ewes, camels, etc.). Of the 46 articles screened in full, 24 articles were included. Two additional papers were identified from screening reference lists, resulting in a total of 26 articles included in the review. Among them, 11 $(42 \%)$ reported the incidence or prevalence of lactational mastitis, five $(19 \%)$ reported risk factors for lactational mastitis, and $10(38 \%)$ included both.

\section{Data Collection}

Six electronic databases (Medline, Embase, CINAHL, Cochrane, Global Health, and Web of Science) were searched in July 2017 using Medical Subject Headings (MeSH) and keyword searches of titles and abstracts. No geographical, population, language, or date of publication limitations were excluded. We searched for a combination of three main concepts: mastitis, burden of disease, and risk factors (see Supplementary Material 1 for more details).

References identified in the search were de-duplicated using EndNote and two authors (EW and LB) independently screened titles and abstracts to identify relevant articles. Two authors (EW and LB) independently screened all full text articles and extracted data using a predefined form in Excel. Differences were resolved through discussion. Data extracted were full reference, study design, definition of mastitis, population in study, time of disease occurrence, risk factors, definition of measure(s) of disease frequency, and risk factor analysis including adjustment for confounders. 
Papers identified in electronic database searching

$(\mathrm{N}=6562)$

Global Health (1296), Embase (2445), Medline (2085), Web of Science (565), Cochrane (48), CINAHL (123)

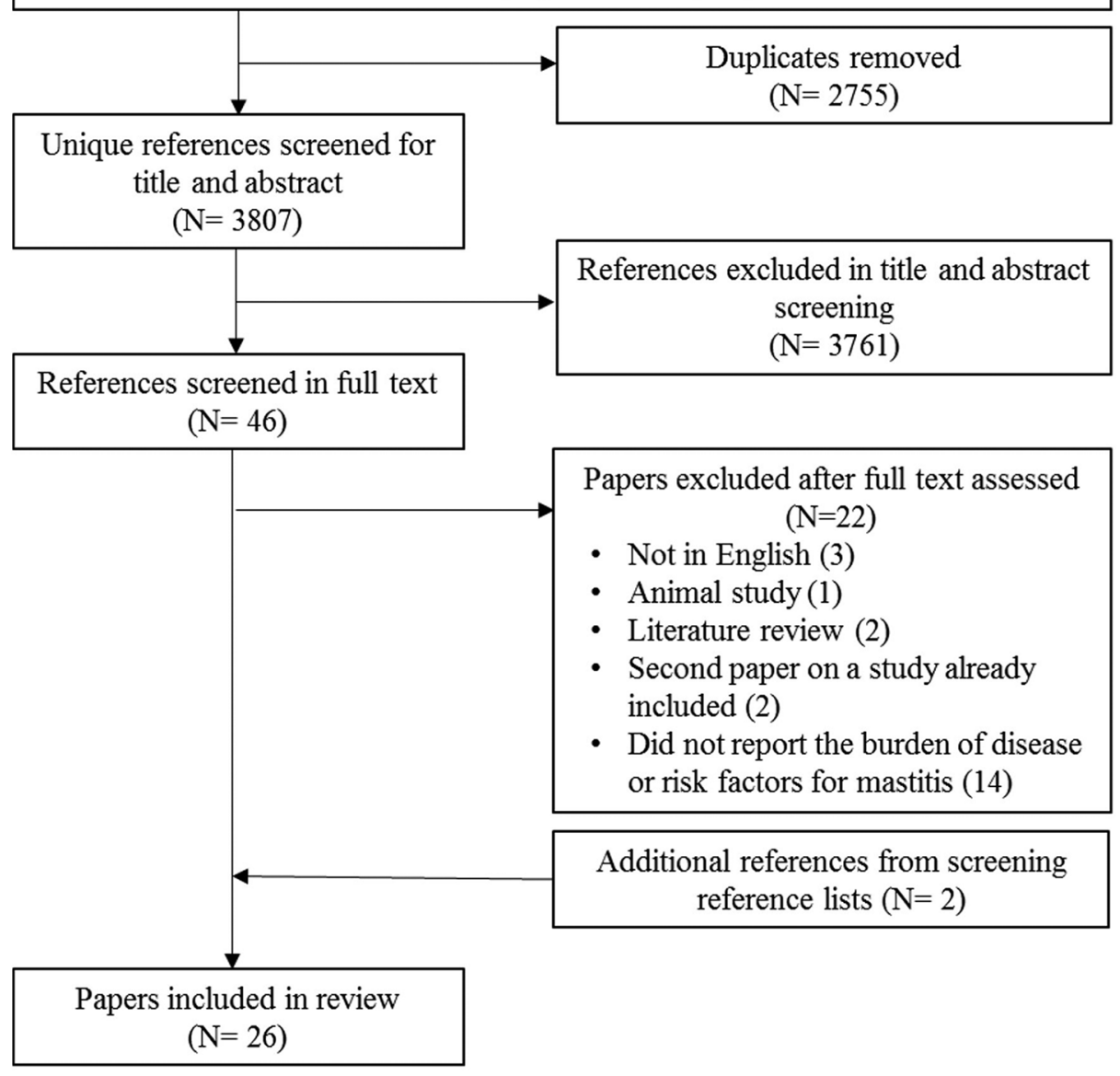

Figure I. Search results flow chart.

\section{Data Analysis}

Study design, measurement and findings of studies were described and synthesized in a narrative format using extracted data. We summarized measures of frequency (several various measures were used by the researchers of reviewed articles) and measures of association between risk factors and mastitis. A meta-analysis of the three studies that measured the incidence of mastitis in an appropriate and comparable manner was conducted. We produced pooled estimates for each 4-week period postpartum, and for the whole period between birth and Week 25 postpartum through the random effects model using the DerSimonian and Laird (1986) method of estimating heterogeneity through inverse variance weighting. Heterogeneity between studies was assessed with a chi-square test (Cochran Q statistic) and quantified with the $I^{2}$ statistic. Due to the very high levels of heterogeneity found $\left(I^{2}>95 \%\right.$ for Week $0-4$ and $>80 \%$ in
Weeks 5-8, 9-12, and 13-16) we chose not to present the summary estimates for each 4-week period postpartum. Rather, one summary estimate of the incidence rate of lactational mastitis for the entire period between birth and 25 weeks postpartum and its $95 \%$ confidence interval is reported. Risk factors assessed by the researchers in reviewed studies were extracted, summarized by broad categories and findings reported according to whether crude or adjusted analysis of each risk factor was conducted. We did not conduct a systematic assessment of quality of included studies.

\section{Results}

\section{Sample Characteristics}

Of the 26 included articles (Table 1), 10 (38\%) were conducted in Australia or New Zealand, seven (27\%) in Europe (one each in Finland, Denmark, Spain, Sweden, and 
Germany, and two in the United Kingdom), four (15\%) in the United States, three (12\%) in Asia (Nepal, China, and Iran), and two (8\%) in Africa (Gambia and Ghana). Except for two (8\%), all articles were published after 1990.

The majority of the included articles, $18(69 \%)$, reported on cohort studies, three $(12 \%)$ described case control studies, four ( $15 \%)$ were cross-sectional designs and one $(4 \%)$ was a community-based open cohort. Two of the case control studies recruited cases from maternity hospitals ( 1 and 8$)$ and one recruited via referral from lactation consultants (17). Two of the cross-sectional studies recruited women attending breastfeeding conferences (15 and 22).

Twenty (77\%) of the articles described studies that collected data about mastitis and associated risk factors from participants via questionnaires, either self-administered or conducted by an investigator (in person or by phone). Five $(19 \%)$ studies solely or partially relied on data from participants medical records or by collecting data during clinical encounters. The remaining study used a combination of laboratory diagnosis using milk cultures and participants' selfreported risk factors. Among the 18 cohort studies, the follow-up period for measuring frequency of lactational mastitis ranged from 6 weeks to 12 months postpartum. Eight (31\%) studies followed participants to 6 months or 26 weeks postpartum and one (4\%) study to 12 months. Nine $(35 \%)$ studies did not provide the time of follow-up or were cross-sectional.

\section{Definition of Mastitis}

Studies varied in their definition of lactational mastitis but most used a combination of common breast and systemic symptoms (Table 1). Breast symptoms included pain, redness, a lump, swelling, or being hot to touch. Systemic symptoms included flu-like symptoms (e.g., fever, chills, aching, and/or headache). Some studies specified that the affected participants must have exhibited symptoms for a minimum time period, for example 12 or $24 \mathrm{hr}$.

\section{Frequency of Disease}

A total of $21(81 \%)$ articles provided an estimate of disease frequency of lactational mastitis (Table 1) with large variation in the definition of numerators and denominators. Numerators included episodes of mastitis in some studies and participants affected by mastitis (regardless of number of episodes) in others. Denominators in several studies included non-breastfeeding participants or modeled estimates of the number of participants expected to be breastfeeding. The reported percentage of participants experiencing mastitis ranged from $2.5 \%$ (article 16 in Table 1 ) to $20 \%$ (articles 4 and 14 in Table 1). Three $(12 \%)$ studies provided an incidence density rate, calculated as the number of mastitis episodes per breastfeeding weeks at risk. Prospective cohort studies carried out in Australia, China, and Iran were conducted among participants who gave birth in hospitals and were followed from immediately after childbirth until week 26 postpartum. These studies defined mastitis identically and utilized similar methods of data collection (telephone interviews with participants). In all three studies, the incidence of mastitis was highest during the first 4 weeks postpartum and then declined dramatically for each of the 4-week intervals thereafter (Figure 2). Despite their methodological homogeneity the reported incidence density rates varied widely across the three studies and $I^{2}$ values were high $(>60 \%)$ in all time periods except weeks 22 25 postpartum. The study in Australia reported the highest incidence at every time-point and the lowest incidence was almost always reported in China. This was most marked during the first 2 months postpartum when reported incidence in Australia was approximately 3 times higher than in China. The pooled incidence rate for the combined period 0-25 weeks postpartum was 11.1 lactational mastitis episodes per 1,000 breastfeeding weeks (95\% CI [10.2-12.0], with a high degree of heterogeneity $\left.\left[I^{2}=98 \%\right]\right)$.

\section{Risk Factors}

Fifteen articles (58\%) reported on analyses of the association of 42 unique risk factors with the occurrence of lactational mastitis. However, most were poorly designed to test specific associations, mainly because they did not have large enough sample sizes, suffered from potential recall and selection bias issues, and were incompletely adjusted for potential confounding. The many factors whose association with lactational mastitis was investigated can be conceptually divided into three categories: factors directly related to anatomical/breastfeeding factors and behaviors, socio-demographic factors, and other factors (Table 2). We extracted the most fully adjusted estimates of association. Due to methodological differences between these studies, we were unable to investigate risk factors using meta-analysis and instead synthesized findings narratively.

Anatomical and Breastfeeding Factors. Nipple damage or nipple pain was the most frequently investigated risk factor for mastitis ( $n=11 ; 42 \%$ of studies). Nipple damage commonly occurs among breastfeeding women during the early postpartum period and can present as cracks, over-dryness, and sores (Spencer, 2008; Wambach, 2003). Nipple damage, operationalized as cracked nipples, was found to be significantly associated with lactational mastitis in all eight studies investigating this association. Researchers examined the association between nipple pain and mastitis in four studies and found a significant and positive association. Presence of other breastfeeding problems, for example attachment difficulties, engorgement, and blocked ducts, were also found to be associated with mastitis.

The use of breastfeeding products was examined as a risk factor for mastitis by several research teams. All those using an adjusted analysis found a significant positive association between the use of various types of nipple creams and 


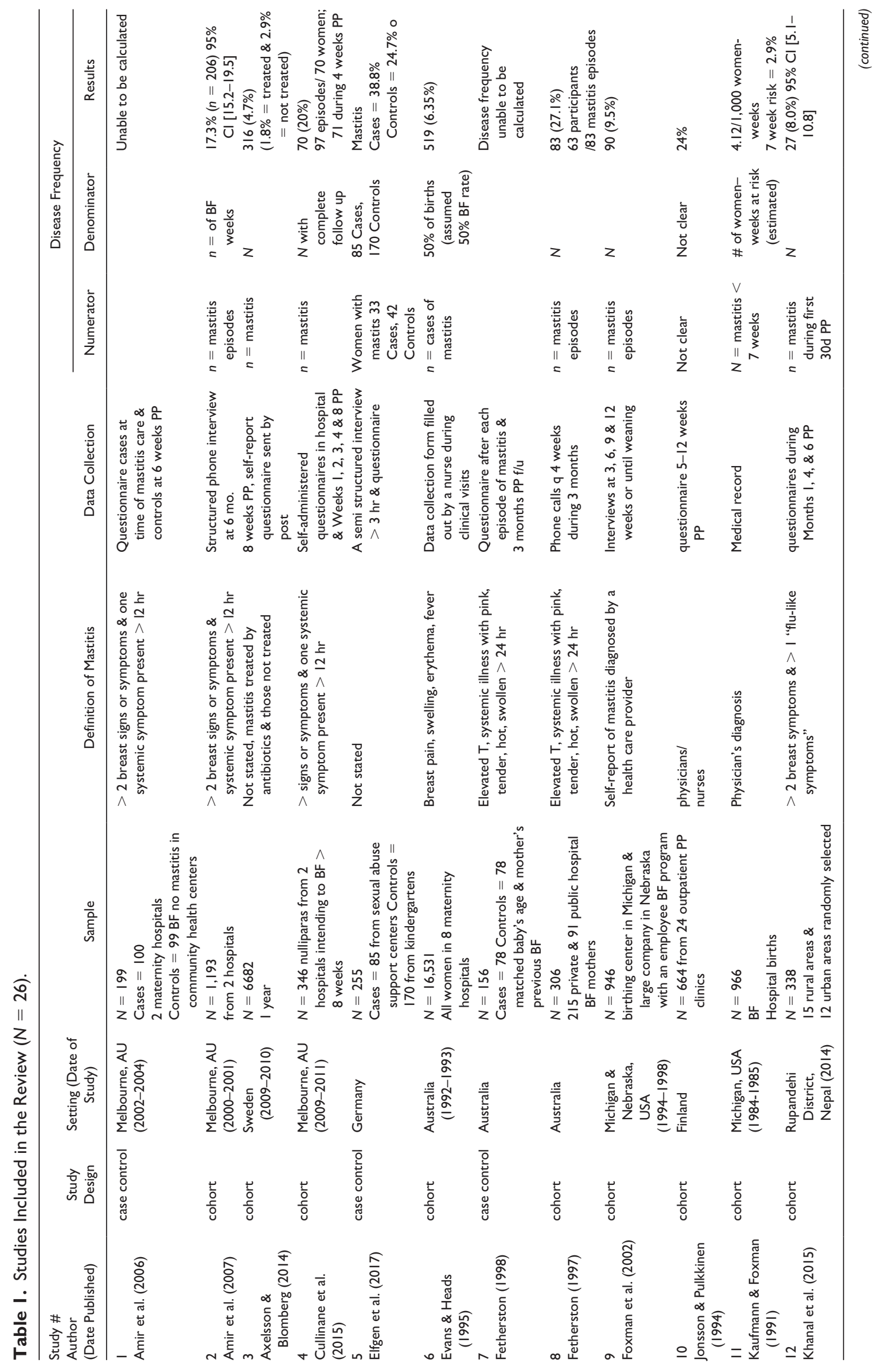




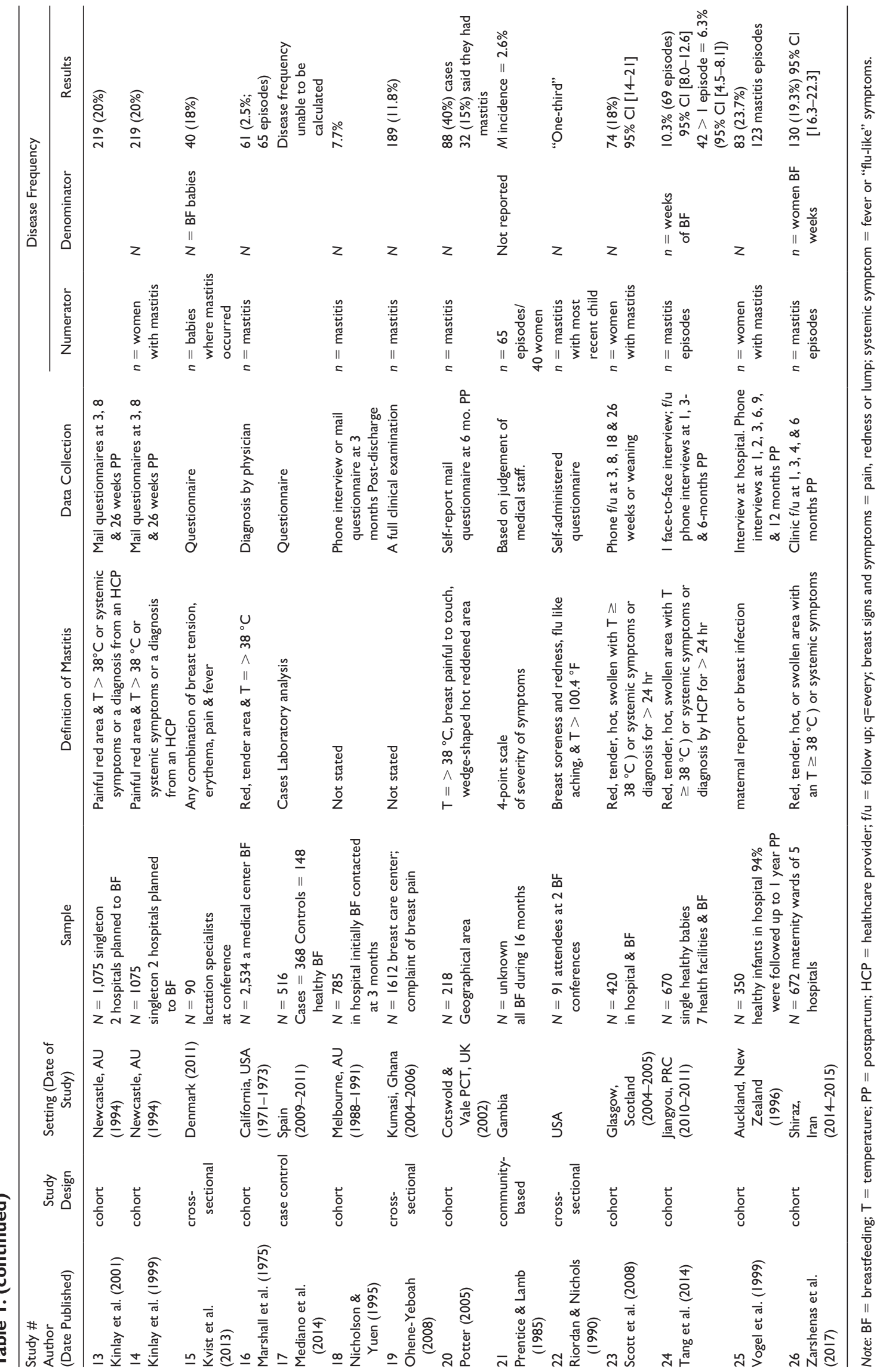




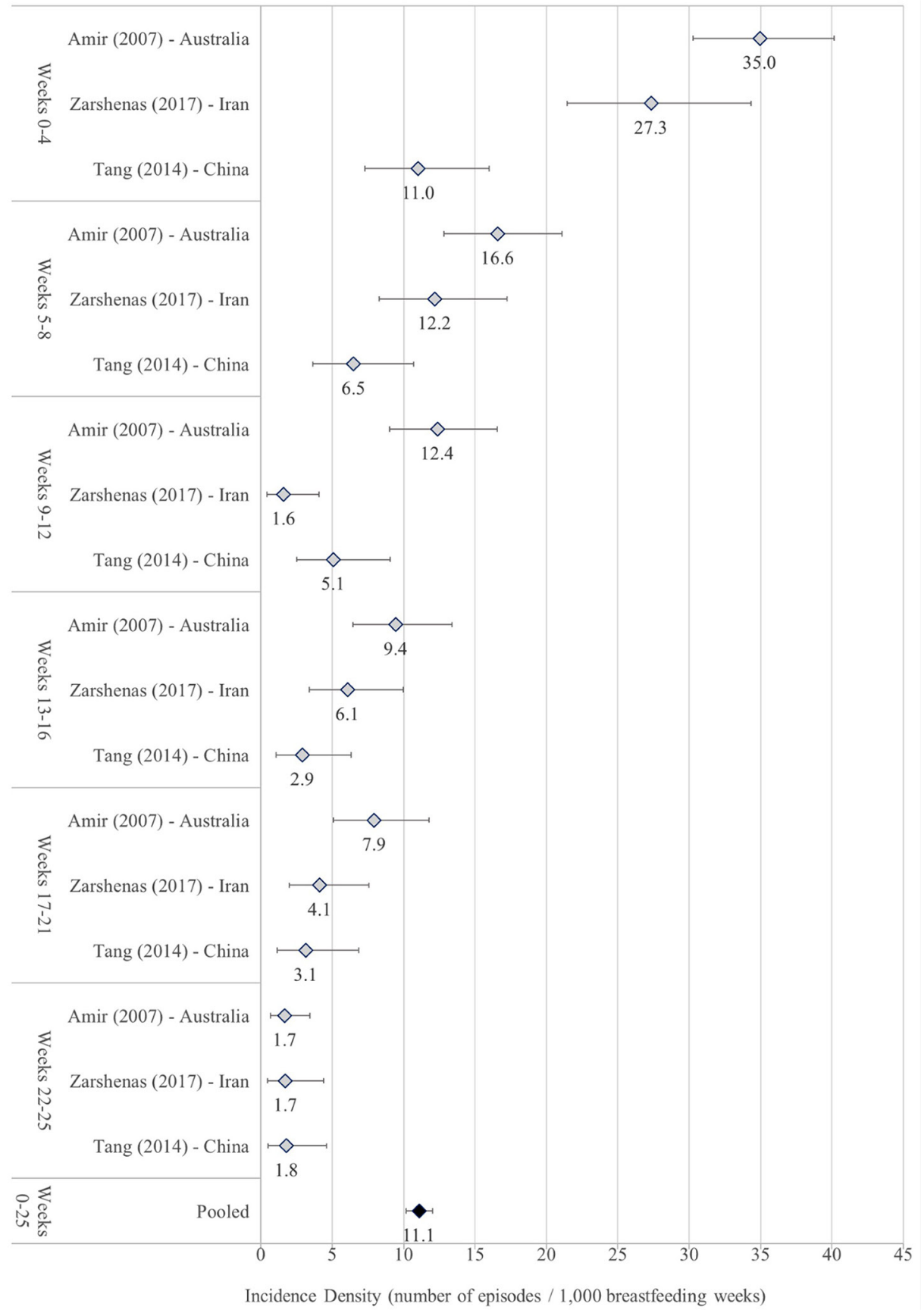

Figure 2. Meta-analysis of incidence density of lactational mastitis by weeks postpartum.

lactational mastitis. One research team found that the use of nipple shields was significantly associated with mastitis. However, it was not always clear whether the use of these products preceded or followed mastitis, and whether their use was precipitated by other potential mastitis risk factors (e.g., cracked nipples), potentially leading to spurious associations due to reverse causality. Various research teams studied timing and frequency of breastfeeding, position, milk supply, expressing milk, and the use of breastfeeding supplements. The evidence on the association between these risk factors and lactational mastitis was variable.

Socio-Demographic Factors. Participants' age was examined as a risk factor for lactational mastitis $(n=5 ; 19 \%)$; however, the findings about the existence of an association and its direction were not consistent. The association with other 


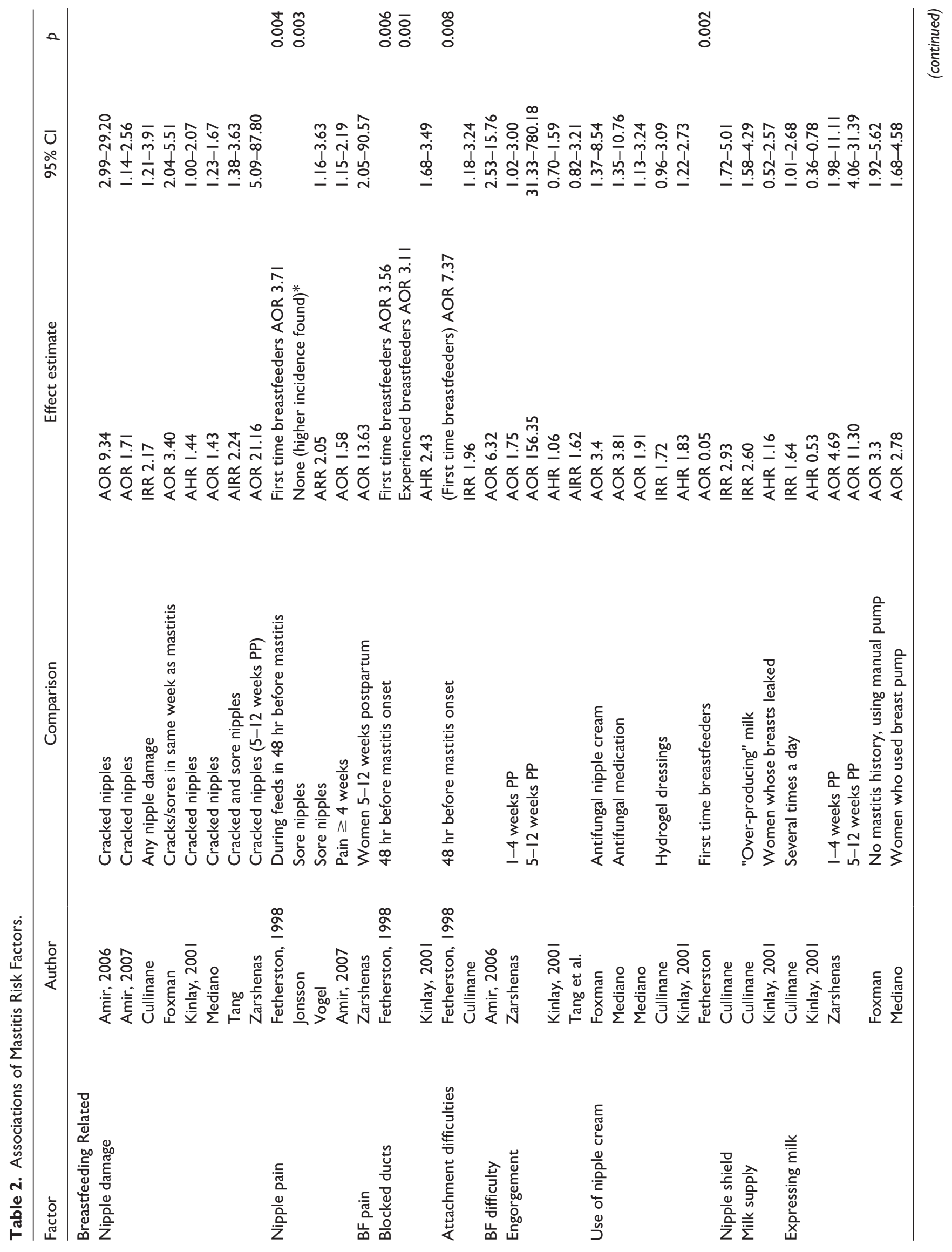




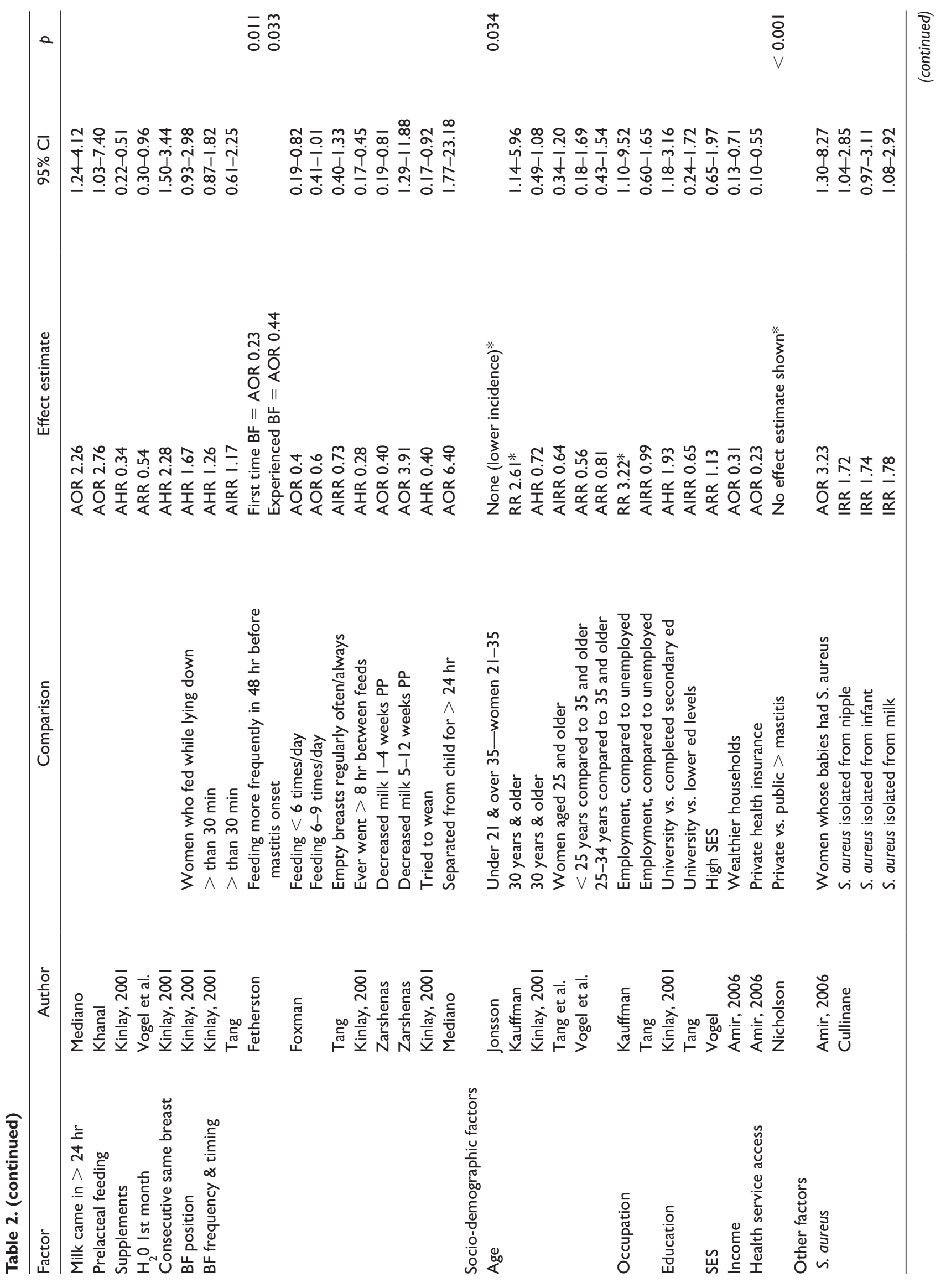




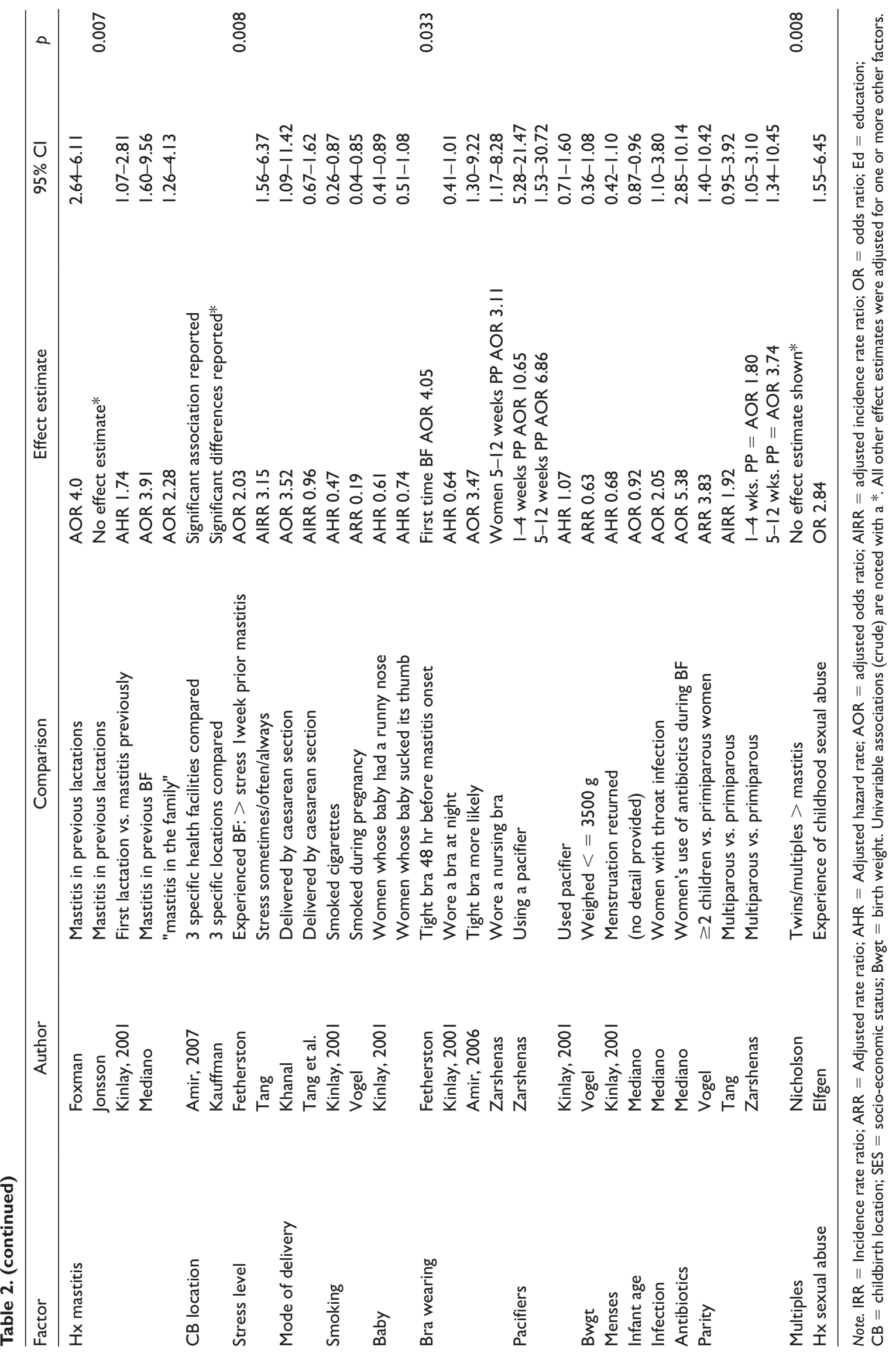


socio-demographic factors, for example education, income, occupation, and access to private care was also mixed.

Other Risk Factors. Another factor investigated by researchers were infections with $S$. aureus. Two (8\%) of the studies reported that participants who carried $S$. aureus on the nipple or had it isolated from their milk, or whose infants carried $S$. aureus, were more likely to have lactational mastitis. Multiple research teams found that women who experienced lactational mastitis during lactation with previous children were significantly more likely to experience mastitis in subsequent lactations. The size of this association ranged from $74 \%$ more likely to 4 times more likely. Three (11\%) studies examined parity as a risk factor in multivariable models. All three found that, compared to primiparous participants, those with two or more children were 2-4 times more likely to have had lactational mastitis, and two of these associations were statistically significant. The use of a tight bra was associated with increased risk of lactational mastitis in three (11\%) studies.

\section{Discussion}

This is the first systematic review to summarize the global evidence on disease frequency and risk factors for lactational mastitis. The epidemiological estimates of disease frequency were variable and mostly low quality, primarily due to unclear denominators including women not at risk for lactational mastitis (not breastfeeding or having recently weaned). However, the three research teams included in the meta-analysis of incidence found a substantial burden of lactational mastitis - with potentially about one in four women breastfeeding up to 25 weeks postpartum affected. The reasons for the large variation in mastitis incidence across the contexts studied are not entirely clear. For the three research teams in meta-analysis, methodological differences might account for some, but relatively little, of these differences. The diagnosis relied on women's responses and is likely to be affected by differences in their expectation, perception, and subsequent reporting of symptoms. However, other factors, including breastfeeding practices and distribution of risk factors, are likely to account for an important proportion of this variation.

Numerous risk factors were assessed and reported to be associated with lactational mastitis. Nipple damage, the most frequently examined risk factor, is common among both experienced and first-time breastfeeding women, and can be caused by attachment difficulties due to improper positioning of infant to breast. Damaged nipples create an entry for pathogens to enter the woman's body, plausibly leading to mastitis. The pathogen commonly associated with acute mastitis is $S$. aureus and infection in the family is associated with the development of mastitis, which may be transferred from women's hands to breast. However, another theory proposes that nipple lesions are a consequence of the inflammatory process of mastitis, rather than a risk factor, introducing the possibility of reverse causality (Contreras,
2011). Breast pumps or nipple shields may contain bacteria and facilitate transfer of infection, thus leading to mastitis, although it is more likely that the association with disease is confounded by the presence of nipple damage, which is a common reason for their use.

Blocked ducts were reported as associated with an increased risk of developing mastitis by numerous researchers. Blocked ducts can cause engorgement, milk stasis, and potentially lead to mastitis. Conversely, the inflammatory process of mastitis may itself cause obstruction, milk stasis, and poor flow, making it hard to untangle the direction of causation (Contreras, 2011). Blocked ducts can occur for a number of reasons, for example a woman missing a feed, attachment difficulties resulting in inadequate milk removal, or wearing tight clothing, all of which are also associated with mastitis (Contreras, 2011). Increased stress levels, professional occupation, and education levels were also associated with increased risk of mastitis in some studies. It is possible that women who are busy with work and family obligations and therefore stressed may be more likely to miss, delay, or cut short a feed, or supplement with formula, all of which may lead to blocked ducts.

History of mastitis in previous lactations was associated with risk of mastitis in multiple studies. Perhaps the aforementioned risk factors continued into subsequent lactations. If a woman is unaware of proper attachment techniques, resulting in nipple damage and mastitis in her first child, it is possible that the same behavior will occur in future lactations. Circumstances, including occupation or life stressors, may not have changed and thus increase the likelihood of mastitis. Alternatively, factors affecting the milk microbiome may persist and important interactions between the woman's immune system and commensal bacteria could increase the risk of infection in each lactation (Contreras, 2011).

Many of the risk factors mentioned in this review appear amenable to mediation by women's breastfeeding behavior and practices. A recent Cochrane review of five randomized controlled trials of interventions for preventing mastitis found no significant difference in mastitis incidence between women who received and did not receive the intervention (Crepinsek et al., 2012). These trials included both use of antibiotics, as well as education and use of breastfeeding techniques to reduce the risk of mastitis; however, the reviewed studies were small and of poor methodological quality. Therefore, determining the effectiveness of these interventions was not possible. This highlights a further gap in research, which can inform clinical practice regarding interventions that are most effective for preventing mastitis.

Relying on retrospective self-reporting of mastitis appeared to be both a strength and limitation of the included studies. It has often been reported that many women do not seek medical attention for mild lactational mastitis and elect to self-manage the condition. With this knowledge, using self-report methods to measure the incidence of mastitis 
would provide more accurate estimates as it minimizes the potential of missing cases compared to using medical records. However, relying on self-reports creates potential for bias in both the measurement of the burden of disease and risk factors for lactational mastitis. While the sensitivity of self-reported physical symptoms for mastitis is likely high due to the traumatic nature of the illness, women's ability to accurately self-report the occurrence and timing of potential risk factors, including blocked ducts or damaged nipples, is questionable and susceptible to recall bias. Although there are some accepted clinical symptoms of mastitis that are generally used for diagnosis, there is no globally accepted standard definition. While the combination of symptoms used across the reviewed studies to define mastitis was similar, a standardized validated tool to collect data about lactational mastitis using women's self-reports would benefit future research about its incidence and risk factors. It would ensure that all researchers measured mastitis in exactly the same manner and increase the comparability and generalizability of findings.

Only three of the included studies were conducted in lowincome countries. It is difficult to say whether the results from this review on incidence of lactational mastitis are generalizable to low-resource settings. Women in these settings may have different cultural norms when it comes to both medical and non-medical support (e.g., informal or familial support networks). There may be cultural differences in breastfeeding practices that have not been explored. There may be differences in maternal gut bacteria leading to different milk microbiomes. Only one study from a low-resource setting (Nepal) examined risk factors for lactational mastitis. It is likely that women in low-income settings have different risk factors than women in high-income settings, including access to breastfeeding products (e.g., nipple shields, breast pumps), but may be exposed to risk factors and protective practices that have not been captured in existing studies. The consequences of lactational mastitis, particularly breastfeeding discontinuation (Wockel et al., 2008), are crucial areas for future research in these settings. The majority of included studies recruited participants from hospitals or community health centers. In some cases, for instance where not all women delivered in a hospital, the sample may not be representative of the total population for various reasons (socioeconomic status, cultural norms, stigma), and therefore reduce the generalizability of the results. However, most studies were conducted in high-income settings (Australia, United States, and Europe) where giving birth in a hospital is accessible and the general sociocultural and medical norm.

There is a wide variation in the estimates of the disease frequency of mastitis, but it is clear that this painful illness is common across a range of settings. We recommend an international collaboration to work on the definition of and measurement tools for mastitis to ensure consistency in future research. Many of the behavioral and physical risk factors for mastitis appear to be preventable but are interdependent. Disentangling their temporal and causal effects on lactational mastitis requires sound epidemiological methods and study designs.

\section{Limitations}

While we applied rigorous search protocols, inclusion and exclusion criteria, and placed no limitations on date, country, or populations of studies, this review has some limitations. We searched six databases but might have missed publications not indexed in these databases, and those for which the abstract or full text was not available in English. We did not conduct a quality assessment of the included studies or contact study authors for clarifications. Last, due to the high heterogeneity of the pooled estimate, caution needs to be exercised in the interpretation of the meta-analysis.

\section{Conclusions}

This review is the first to summarize global evidence of the burden of disease and risk factors for lactational mastitis. The need for high-quality research is particularly high in low-resource settings due to a paucity of evidence and the disproportionately negative consequences of the illness and of breastfeeding discontinuation on both infants and women's health.

\section{Declaration of Conflicting Interests}

The authors declared no potential conflicts of interest with respect to the research, authorship, and/or publication of this article.

\section{Funding}

The authors received no financial support for the research, authorship, and/or publication of this article.

\section{ORCID iDs}

Susannah L Woodd (iD https://orcid.org/0000-0001-7389-2351

Lenka Benova (iD https://orcid.org/0000-0001-8595-365X

\section{Supplemental Material}

Supplementary Material may be found in the "Supplemental material" tab in the online version of this article.

\section{References}

Amir, L. H., Forster, D. A., Lumley, J., \& McLachlan, H. (2007). A descriptive study of mastitis in Australian breastfeeding women: Incidence and determinants. BMC Public Health, 7, Article 62. doi:10.1186/1471-2458-7-62

Amir, L. H., Garland, S. M., \& Lumley, J. (2006). A case-control study of mastitis: Nasal carriage of Staphylococcus aureus. BMC Family Practice, 7, Article 57. doi:10.1186/1471-2296-7-57

Axelsson, D., \& Blomberg, M. (2014). Prevalence of postpartum infections: A population-based observational study. Acta Obstetricia et Gynecologica, 93(10), 1065-1068. 
Baeza, C. (2016). Acute, Subclinical, and Subacute Mastitis: Definitions, Etiology, and Clinical Management. Clinical Lactation, 7(1), 7-10.

Crepinsek, M. A., Crowe, L., Michener, K., \& Smart, N. A. (2012). Interventions for preventing mastitis after childbirth. Cochrane Database of Systematic Reviews. doi: 10.1002/14651858. CD007239.pub3

Contreras, G. A., \& Rodriguez, J. M. (2011). Mastitis: comparative etiology and epidemiology. Journal of Mammary Gland Biology and Neoplasia, 16(4), 339-356.

Cullinane, M., Amir, L. H., Donath, S. M., Garland, S. M., Tabrizi, S. N., \& Payne, M. S. (2015). Determinants of mastitis in women in the CASTLE study: A cohort study. BMC Family Practice, 16, Article 181. https://doi.org/10.1186/s12875-0150396-5.

Delgado, S., Arroyo, R., Martín, R., \& Rodríguez, J. M. (2008). PCR-DGGE assessment of the bacterial diversity of breast milk in women with lactational infectious mastitis. BMC Infectious Diseases, 8, Article 51. https://doi. org/10.1186/1471-2334-8-51

DerSimonian, R., \& Laird, N. (1986). Meta-analysis in clinical trials. Controlled Clinical Trials, 7(3), 177-188.

Dieterich, C., Felice, J., O'Sullivan, E., \& Rasmussen, K. (2013). Breastfeeding and health outcomes for the mother-infant dyad. Pediatric Clinics of North America, 60(1), 31-48.

Elfgen, C., Hagenbuch, N., Görres, G., Block, E., \& Leeners, B. (2017). Breastfeeding in Women Having Experienced Childhood Sexual Abuse. Journal of Human Lactation, 33(1), 119-127.

Evans, M., \& Heads, J. (1995). Mastitis: Incidence, prevalence and cost. Breastfeeding Review, 3(2), 65-72.

Fetherston, C. (1997) Characteristics of lactation mastitis in a Western Australian cohort. Breastfeeding Review, 5(2), 5-11.

Fetherston, C. (1998). Risk factors for lactation mastitis. Journal of Human Lactation, 14(2), 101-109.

Foxman, B., D’Arcy, H., Gillespie, B., Bobo, J. K., \& Schwartz, K. (2002). Lactation mastitis: Occurrence and medical management among 946 breastfeeding women in the United States. American Journal of Epidemiology, 155(2), 103-114.

Gon, G., Leite, A., Calvert, C., Woodd, S., Graham, W. J., \& Filippi, V. (2018). The frequency of maternal morbidity: A systematic review of systematic reviews. International Journal of Gynecology and Obstetrics, 141 (Suppl. 1), 20-38.

Hager, W. D., \& Barton, J. R. (1996). Treatment of sporadic acute puerperal mastitis. Infectious Diseases in Obstetrics and Gynecology, 4(2), 97-101.

Jonsson, S., \& Pulkkinen, M. O. (1994). Mastitis today: Incidence, prevention, and treatment. Annales Chirurgiae et Gynaecologiae, 83(208), 84-87.

Kaufmann, R., \& Foxman, B. (1991). Mastitis among lactating women: Occurrence and risk factors. Social Science and Medicine, 33(6), 701-705.

Khanal, V., Scott, J. A., Lee, A. H., \& Binns, C. (2015). Incidence of mastitis in the neonatal period in a traditional breastfeeding society: Results of a cohort study. Breastfeeding Medicine, 10(10), 481-487.

Kinlay, J. R., O’Connell, D. L., \& Kinlay, S. (2001). Risk factors for mastitis in breastfeeding women: Results of a prospective cohort study. Australian and New Zealand Journal of Public Health, 25(2), 115-120.
Kinlay, J. R., O’Connell, D. L., \& Kinlay, S. (1999). Incidence of mastitis in breastfeeding women during the six months after delivery: A prospective cohort study. Obstetrical and Gynecological Survey, 54(4), 218-219.

Kvist, L. J., Larsson, B. W., Hall-Lord, M. L., Steen, A., \& Schalén, C. (2008). The role of bacteria in lactational mastitis and some considerations of the use of antibiotic treatment. International Breastfeeding Journal, 3(6). doi: 10.1186/1746-4358-3-6.

Kvist, L. J. (2013). Re-examination of old truths: Replication of a study to measure the incidence of lactational mastitis in breastfeeding women. International Breastfeeding Journal, 8(2). doi: 10.1186/1746-4358-8-2.

Lawrence, R. (1989). Breastfeeding: A guide for the medical profession. Mosby.

Marín, M., Arroyo, R., Espinosa-Martos, I., Fernández, L., \& Rodríguez, J. M. (2017). Identification of emerging human mastitis pathogens by MALDI-TOF and assessment of their antibiotic resistance patterns. Frontiers in Microbiology, 8(1258). doi: 10.3389/fmicb.2017.01258.

Marshall, B. R., Hepper, J. K., \& Zirbel, C. C. (1975). Sporadic puerpural mastitis. An infection that need not interrupt lactation. Journal of the American Medical Association, 233(13), 1377-1379.

Mediano, P., Fernández, L., Rodríguez, J. M., \& Marín, M. (2014). Case-control study of risk factors for infectious mastitis in Spanish breastfeeding women. BMC Pregnancy and Childbirth, 14(195). doi: 10.1186/1471-2393-14-195.

Michie, C., Lockie, F., \& Lynn, W. (2003). The challenge of mastitis. Archives of Disease in Childhood, 88(9), 818-821.

Nicholson, W., \& Yuen, H. P. (1995). A study of breast feeding rates at a large Australian obstetric hospital. Australian and New Zealand Journal of Obstetrics and Gyneacology, 35(4), 393-397.

Ohene-Yeboah, M. (2008). Breast pain in Ghanaian women: Clinical, ultrasonographic, mammographic and histological findings in 1612 consecutive patients. West African Journal of Medicine, 27(1), 20-23.

Osterman, K. L., \& Rahm, V. (2000). Lactation mastitis: Bacterial cultivation of breast milk, symptoms, treatment, and outcome. Journal of Human Lactation, 16(4), 297-302.

Potter, B. (2005). A multi-method approach to measuring mastitis incidence. Community practitioner, 78(5),169-173.

Prentice, A., \& Lamb, W. (1985). Mastitis in rural Gambian mothers and the protection of the breast by milk antimicrobial factors. Transactions of the Royal Society of Tropical Medicine and Hygiene, 79(1), 90-95.

Riordan, J. M., \& Nichols, F. H. (1990). A descriptive study of lactation mastitis in long-term breastfeeding women. Journal of Human Lactation, 6(2), 53-58.

Scott, J. A., Robertson, M., Fitzpatrick, J., Knight, C., \& Mulholland, S. (2008). Occurrence of lactational mastitis and medical management: A prospective cohort study in Glasgow. International Breastfeeding Journal, 3(21). doi: 10.1186/1746-4358-3-21.

Spencer, J. (2008). Management of mastitis in breastfeeding women. American Family Physician, 78(6), 727-731.

Tang, L., Lee, A. H., Qiu, L., \& Binns, C. W. (2014). Mastitis in Chinese breastfeeding mothers: A prospective cohort study. Breastfeeding Medicine, 9(1), 35-38.

Thomsen, A. C., Espersen, T., \& Maigaard, S. (1984). Course and treatment of milk stasis, noninfectious inflammation of the 
breast, and infectious mastitis in nursing women. American Journal of Obstetrics \& Gynecology, 149(5), 492-495.

Vogel, A., Hutchison, B. L., \& Mitchell, E. A. (1999). Mastitis in the first year postpartum. Birth: Issues in Perinatal Care, 26(4), 218-225.

Wambach, K. A. (2003). Lactation mastitis: A descriptive study of the experience. Journal of Human Lactation, 19(1), 24-34.

Wockel, A., Abou-Dakn, M., Beggel, A., \& Arck, P. (2008). Inflammatory breast diseases during lactation: Health effects on the newborn-a literature review. Mediators of Inflammation. doi: $10.1155 / 2008 / 298760$.

World Health Organization. (2000). Mastitis: Causes and management. http://apps.who.int/iris/bitstream/10665/66230/1/WHO_ FCH_CAH_00.13_eng.pdf

Zarshenas, M., Zhao, Y., Poorarian, S., Binns, C. W., Scott, J. A., \& Zhao, Y. (2017). Incidence and risk factors of mastitis in Shiraz, Iran: Results of a cohort study. Breastfeeding Medicine, 12(5), 290-296. 